\title{
Secondary pulmonary conventional chordoma arising from primary sarcomatoid chordoma of the sacrum: A case report
}

\author{
JIA-HONG CHEN ${ }^{1}$, KUAN-YU CHEN ${ }^{2}$, DUENG-YUAN HUENG ${ }^{3}$ and JONG-SHIAW JIN ${ }^{4}$ \\ ${ }^{1}$ Department of Medicine, Division of Hematology/Oncology, Tri-Service General Hospital, National Defense Medical Center, \\ Taipei 114; ${ }^{2}$ Hualien Armed Forces General Hospital, Hualien 920; Departments of ${ }^{3}$ Neurological Surgery and ${ }^{4}$ Pathology, \\ Tri-Service General Hospital, National Defense Medical Center, Taipei 114, Taiwan, R.O.C.
}

Received September 13, 2013; Accepted March 14, 2014

DOI: $10.3892 / 01.2014 .2100$

\begin{abstract}
Chordomas are low- to intermediate-grade malignant tumors that recapitulate the notochord. Chordomas belong to the dysontogenetic bone tumors and appear primarily in the region of the axial skeleton. Chordomas are divided into conventional, chondroid, sarcomatoid and dedifferentiated subtypes. The different subtypes of chordoma have varied survival periods. According to the literature to date, secondary pulmonary and lymph-node metastases occur most frequently, followed by liver, bone and skin metastases. To the best of our knowledge, there has been no previous report of one subtype of chordoma metastasizing or transforming into another subtype with a different histopathology. This study presents a 24-year-old man with secondary pulmonary conventional chordoma arising from a primary sarcomatoid chordoma of the sacrum. The patient was alive at the end of November, 2009 and the survival time exceeded eight years. This is the first case of a patient with primary sarcomatoid chordoma of the sacrum with complete remission in whom a secondary pulmonary conventional chordoma arose from the primary cancer.
\end{abstract}

\section{Introduction}

Chordomas are low- to intermediate-grade malignant tumors that recapitulate the notochord. Etiologically, chordomas belong to the dysontogenetic bone tumors and occur predominantly in the region of the clivus (1). Corresponding to their course of embryological development, chordomas primarily appear in the region of the axial skeleton and typically occur in midlife, from the ages of 40 to 60 years, and primarily affect men (2).

Correspondence to: Dr Jong-Shiaw Jin, Department of Pathology, Tri-Service General Hospital, National Defense Medical Center, No. 325, Section 2 Cheng-Kung Road, Neihu, Taipei 114, Taiwan, R.O.C.

E-mail: kuanyu8514498000@yahoo.com.tw

Key words: chordoma, sarcomatoid, conventional, transformation
The histological appearance of classical chordoma is of a lobulated tumor composed of groups of cells separated by fibrous septa. The cells have small round nuclei and abundant vacuolated cytoplasm, occasionally described as physaliferous (having bubbles or vacuoles) (3).

Although chordomas are locally invasive, metastasis has been reported in $10-42 \%$ of cases. However, these may be the result of the misdiagnosis of mucus-producing carcinomas of the rectum as chordomas in certain case studies (4-6). Previous studies suggest that secondary pulmonary and lymph-node metastases occur most frequently, followed by liver, bone and skin metastases $(4,7)$.

According to the current literature, no case of a chordoma subtype that has metastasized or transformed into another subtype with a different histopathology and immunoreactivity has been previously reported. This study presents a case of secondary pulmonary conventional chordoma arising from a primary sarcomatoid chordoma of the sacrum.

\section{Case report}

A 24-year-old man was referred to the Tri-Service General Hospital (Taipei, Taiwan, R.O.C) presenting with a palpable sacral mass and constipation of two months in duration. A magnetic resonance imaging (MRI) examination of the sacral spine revealed a large mass (measuring $13 \times 8 \times 7 \mathrm{~cm}$ ) with compression of the colon (Fig. 1A). Based on the clinical and radiological characteristics, the patient underwent an exploratory laparotomy with debulking of the tumor. The gross findings of the sacral tumor included multilobulated, soft, gelatinous masses with deceptively discrete margins. Microscopic analysis revealed a lobular architecture, spindle tumor cells with eosinophilic vacuolated cytoplasm and a mucoid matrix. The pathological diagnosis was a sarcomatoid-type chordoma. Immunohistochemical staining of the sacral tumor was positive for cytokeratin (CK), epithelial membrane antigen (EMA), vimentin, S100 and periodic acid-Schiff (Fig 2A and B). Postoperative adjuvant radiotherapy (50 Gy) was performed.

After one year, the patient presented to the Tri-Service General Hospital with dizziness and unstable gait of one week in duration. Computed tomography $(\mathrm{CT})$ of the brain revealed a mass lesion over the right cerebellar vermis. A suboccipital 
A

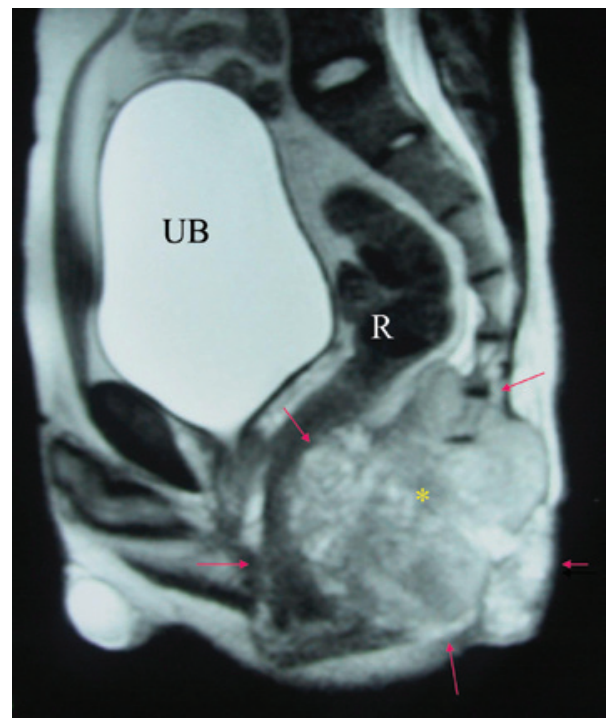

B

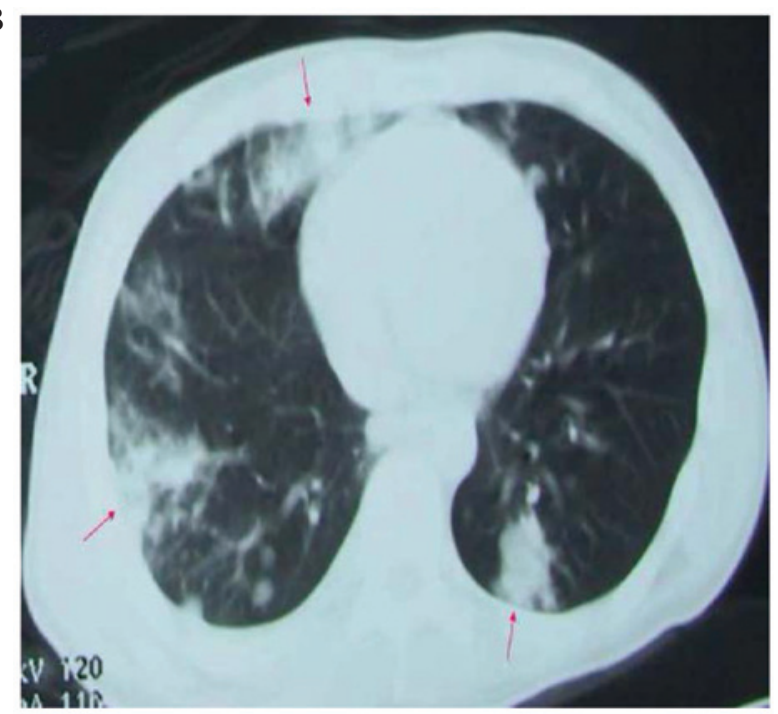

Figure 1. (A) Gadolinium contrast-enhanced magnetic resonance imaging shows a large tumor at the sacrum, with compression of the intestine and urinary bladder. The pink arrows indicate the tumor edge and the asterisk indicates the center of the tumor. (B) Multiple metastases in the bilateral lung fields. The pink arrows indicate the the tumor edge.
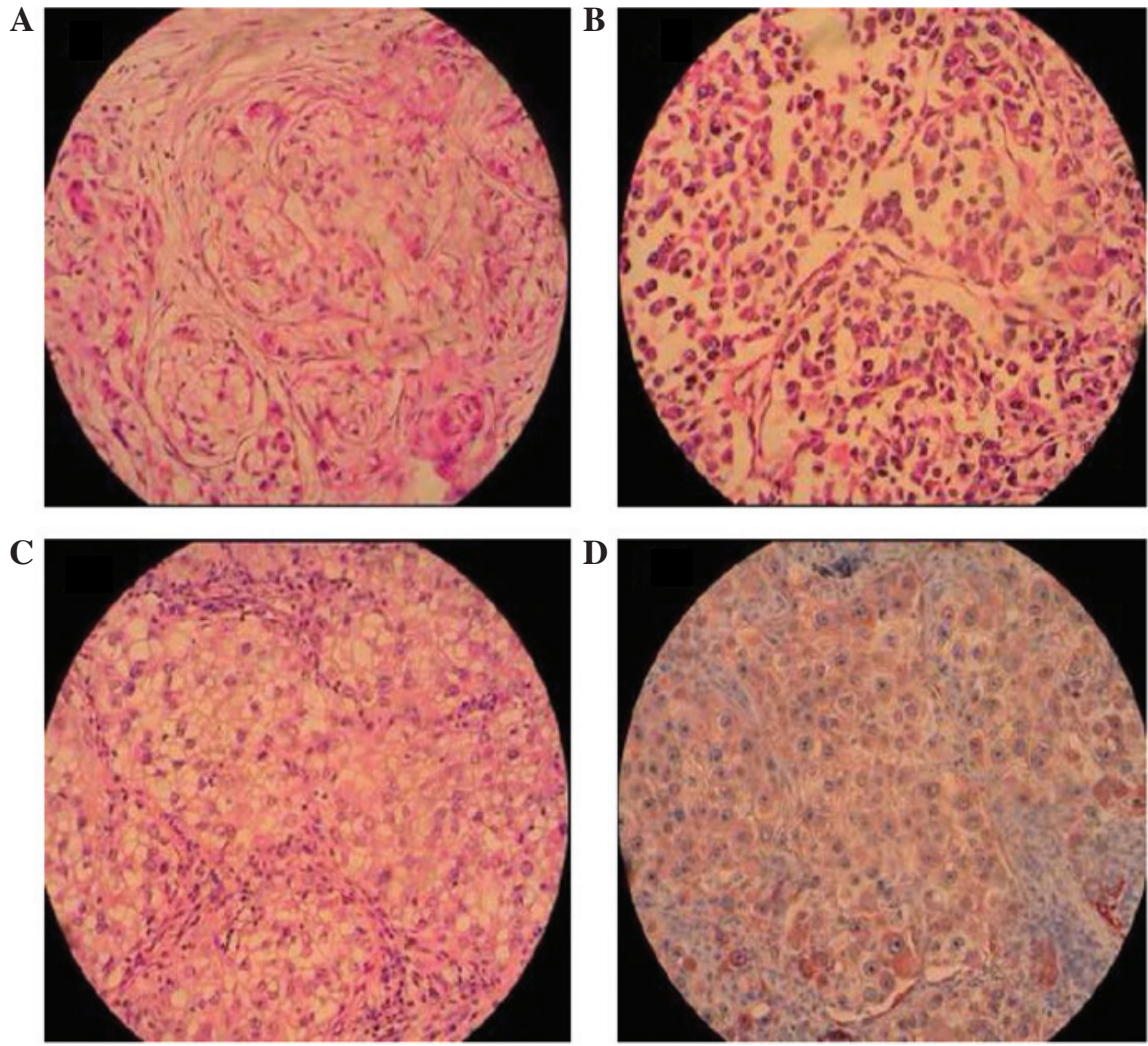

Figure 2. Two different subtypes of chordoma identified in one patient. (A) Histopathology of the conventional chordoma of the sacrum revealed a lobular architecture, eosinophilic vacuolated (physaliphorous) cytoplasm and a mucoid matrix (H\&E stain). (B) Histopathology of the sarcomatoid chordoma of the sacrum revealed a lobular architecture with spindle tumor cells, eosinophilic vacuolated (physaliphorous) cytoplasm and a mucoid matrix (H\&E stain). (C) Histopathology of the conventional chordoma of the lung revealed a lobular architecture, eosinophilic vacuolated (physaliphorous) cytoplasm and a mucoid matrix (H\&E stain). (D) Immunohistochemistry of the conventional chordoma of the lung was positive for cytokeratin (cytokeratin stain). Original magnification, $x 100$. H\&E, hematoxylin and eosin.

craniotomy was performed, with removal of the mass and the placement of a ventriculoperitoneal shunt. The pathological findings identified a sarcomatoid-type metastatic chordoma. Immunohistochemical staining was positive for CK, EMA, vimentin and S100. Intracranial whole-brain radiotherapy and adjuvant chemotherapy with methotrexate were administered to the patient.

After two years, a CT examination of the lungs was performed due to a chronic cough, revealing multiple lesions, enlarged lymph nodes and suspected metastases over the right 
lower lobe (Fig. 1B). A thoracotomy with a wedge resection of the right lower lobe was performed. The final histopathological evaluation of the lung tumor tissue following an immunohistochemical examination revealed a metastatic conventional chordoma (Fig. 2C and D). Cisplatin-based chemotherapy was administered and the disease was stable after four courses of treatment. However, disease progression was noted after six months and persistent salvage chemotherapy was administered. The patient remained alive at the end of November, 2009 and, thus, the survival time had exceeded eight years.

\section{Discussion}

In the present case, the patient suffered from two different subtypes of chordoma. The first was a sarcomatoid chordoma that responded completely to an exploratory laparotomy with debulking of the tumor and postoperative radiotherapy, with no evidence of residual disease. The secondary chordoma was the conventional subtype. To the best of our knowledge, this study is the first to present the case of a different subtype of chordoma arising at a metastatic site.

The majority of patients with conventional-type chordoma are 50-70 years old and 50\% of these tumors arise in the sacrococcygeal region (8). The pathological findings of conventional chordoma include sheet- or cord-like tumor cells floating in the myxoid stroma, with abundant vacuolated cytoplasm (physaliphorous cells). The mean survival is 4.1 years. In sarcomatoid chordomas, the tumor cells display a storiform architecture with large, pleomorphic nuclei. A transitional characteristic distinguishing sarcomatoid chordoma from conventional chordoma is positive CK immunoreactivity in the sarcomatoid component, which is required for a pathological diagnosis (9-12). Prognosis of chordoma is associated with the extent of surgical removal; a five-year survival of $35 \%$ has been reported with incomplete resection if followed by conventional radiation therapy (13).

In the dedifferentiated type of chordoma, the pathological findings show a sharp demarcation between the conventional chordoma and the high-grade sarcoma, with no transitional characteristics between the two components, and negative CK immunoreactivity in the sarcoma component (14-16).

The primary subtype of the chordoma affects the survival period and the progressive characteristics of the tumor, including local relapses, surgical pathway seeding and progressive distal metastasis (5-43\% of patients develop metastatic tumors in the skin, subcutaneous tissue, bones, lungs and lymph nodes) (17). Therefore, the subtype of a metastatic lesion correlates with its histological differentiation and the duration of the clinical course.

Standard treatments for conventional chordoma are maximal surgical resection and subsequent radiotherapy (50 Gy), even proton-beam therapy. These treatments may improve morbidity and mortality (18). However, a two-year mean overall survival period has previously been reported for hematogenous metastases that include a sarcomatous component (19). The patient in the present case was alive at the end of 2009, despite disease progression with metastases. In summary, to the best of our knowledge, this study presents the first patient with primary sarcomatoid chordoma of the sacrum with complete remission, in whom a secondary pulmonary conventional chordoma arose from the primary tumor.

\section{References}

1. Wright D: Nasopharyngeal and cervical chordoma-some aspects of their development and treatment. J Laryngol Otol 81: 1337-1355, 1967.

2. Jenny J and Sulser H: Metastazing chordoma of the lumbar spine. Schweiz Med Wochenschr 103: 697-701, 1973 (In German).

3. Chugh R, Tawbi H, Lucas DR, Biermann JS, Schuetze SM and Baker LH: Chordoma: the nonsarcoma primary bone tumor. Oncologist 12: 1344-1350, 2007.

4. Chambers PW and Schwinn CP: Chordoma. A clinicopathologic study of metastasis. Am J Clin Pathol 72: 765-776, 1979.

5. Dahlin DC and MacCarthy CS: Chordoma. Cancer 5: 1170-1178, 1952.

6. Higinbotham NL, Phillips RF, Farr HW and Hustu HO: Chordoma. Thirty-five-year study at Memorial Hospital. Cancer 20: 1841-1850, 1967.

7. Su WP, Louback JB, Gagne EJ and Scheithauer BW: Chordoma cutis: a report of nineteen patients with cutaneous involvement of chordoma. J Am Acad Dermatol 29: 63-66, 1993.

8. Unni KK (ed): Chordoma. In: Dahlin's Bone Tumors: General Aspects and Data on 11,087 cases. 5th edition. Lippincott-Raven, New York, NY, pp291-305, 1996.

9. Gay E, Sekhar LN, Rubinstein E, Wright DC, Sen C, Janecka IP and Snyderman $\mathrm{CH}$ : Chordomas and chondrosarcomas of the cranial base: results and follow-up of 60 patients. Neurosurgery 36: 887-897, 1995.

10. Hug EB, Loredo LN, Slater JD, DeVries A, Grove RI, Schaefer RA, Rosenberg AE and Slater JM: Proton radiation therapy for chordomas and chondrosarcomas of the skull base. J Neurosurg 91: 432-439, 1999.

11. Forsyth PA, Cascino TL, Shaw EG, Scheithauer BW, O'Fallon JR, Dozier JC and Piepgras DG: Intracranial chordomas: a clinicopathological and prognostic study of 51 cases. J Neurosurg 78: 741-747, 1993.

12. O'Connell JX, Renard LG, Liebsch NJ, et al: Base of skull chordoma. A correlative study of histologic and clinical features of 62 cases. Cancer 74: 2261-2267, 1994.

13. Zorlu F, Gürkaynak M, Yildiz F, Oge K and Atahan IL: Conventional external radiotherapy in the management of clivus chordomas with overt residual disease. Neurol Sci 21: 203-207, 2000.

14. Hruban RH, May M, Marcove RC and Huvos AG: Lumbo-sacral chordoma with high-grade malignant cartilaginous and spindle cell components. Am J Surg Pathol 14: 384-389, 1990.

15. Miettinen M, Karaharju E and Järvinen $\mathrm{H}$ : Chordoma with a massive spindle-cell sarcomatous transformation. A light- and elctron-microscopic and immunohistochemical study. Am J Surg Pathol 11: 563-570, 1987.

16. Meis JM, Raymond AK, Evans HL, Charles RE and Giraldo AA: 'Dedifferentiated' chordoma. A clinicopathologic and immunohistochemical study of three cases. Am J Surg Pathol 11: 516-525, 1987.

17. Berven S, Zurakowski D, Mankin HJ, Gebhardt MC, Springfield DS and Hornicek FJ: Clinical outcome in chordoma: utility of flow cytometry in DNA determination. Spine (Phila Pa 1976) 27: 374-379, 2002.

18. Ikeda H, Honjo J, Sakurai H, Mitsuhashi N, Fukuda T and Niibe H: Dedifferentiated chordoma arising in irradiated sacral chordoma. Radiat Med 15: 109-111, 1997.

19. Saito A, Hasegawa T, Shimoda T, Toda G, Hirohashi S, Tajima G and Moriya Y: Dedifferentiated chordoma: a case report. Jpn J Clin Oncol 28: 766-771, 1998. 